\title{
Response of antioxidant status in kidney of pigs exposed to aflatoxin B1 to dietary grape seed meal
}

\author{
Laurentiu Mihai Palade ${ }^{1}{ }^{*}$, Ana-Maria Pertea ${ }^{1}$, Ionelia Taranu ${ }^{1}$ \\ ${ }^{*}$ Corresponding author: mihai.palade@ibna.ro
}

${ }^{1}$ National Research Development Institute for Animal Biology and Nutrition, IBNA Balotesti, 077015, Ilfov, Romania.

\section{ABSTRACT}

This study investigated the effects of dietary inclusion of grape seed meal (GSM) on antioxidant markers in kidney of aflatoxin B1 fed pigs. Twenty-four cross-bred pigs (TOPIG-40) were randomly assigned to one of the four experimental diets: control (Control), aflatoxin B1 (diet contaminated with $320 \mu \mathrm{g}$ AFB1/kg feed), grape seed meal (diet with 8\% GSM) and AFB1+GSM (320 $\mu \mathrm{g}$ AFB1/kg feed $+8 \%$ GSM) for 30 days. Aflatoxin B1 induced oxidative damage and showed lower antioxidant status in kidney after 30 days. CAT and SOD activity measured in kidneys revealed significant differences when comparing the GSM+AFB1 group with the AFB1 group. Additionally, GSM diet alone did not affect CAT and SOD levels in kidneys of piglets compared with the control group, suggesting that they are maintained at physiological levels. We did not detect any effects of GSM on GPx and TAS. Including GSM into piglets' diet significantly lowered lipid peroxidation induced by AFB1, while it did not affect it compared with the control group. The present study demonstrated the ability of GSM to alleviate the effects induced by the toxin evidenced by the improvement in the antioxidant status, and suggests the potential applicability of GSM as mycotoxin adsorbent in animal feed.

Keywords: B1, piglets, inflammation, oxidative stress, grape seed meal.

\section{INTRODUCTION}

Widely studied, mycotoxins are fungal toxic secondary metabolites that occur naturally (Colović et al., 2019; Kosicki et al., 2016). Although not entirely elucidated, it is believed that their physiological role in the fungal metabolism is a proliferative aid (Fox and Howlett, 2008).

Aflatoxins (AFs), among the most toxic and health threatening, are mycotoxins mainly produced by Aspergilus flavus, Aspergilus parasiticus and Aspergillus nomius (Kos et al., 2013). They represent a major threat and a 
health concern in animals and humans (Kosicki et al., 2016; Prapapanpong et al., 2019), as contamination with these toxins has increasingly impacted food and feed quality, as well as safety during the past years due to climate change (Battilani et al., 2016).

Aflatoxin B1 is the most toxic among aflatoxins, and it's ingestion through food and feed is regarded as the main way of exposure (Kos et al., 2020). It has been reported that the mycotoxin presence in diets affects the relative weight of liver, kidneys and heart in pigs (Andretta et al., 2012). Moreover, the exposure to high concentrations of AFB1 induces damage to vital organs, subsequently developing into chronic disease (Marin et al., 2019). Besides the liver, spleen, and intestine, the kidney is another key organ afflicted by AFB1 contamination (Andretta et al., 2012; Peles et al., 2019). Literature data pertaining AFB1 toxic effects on kidney are limited. However, it was reported that exposure to AFB1 activated oxidative stress and resulted in renal damage in a 35-day subacute toxicity mice model (Li et al., 2018). As the excretion of AFB1 occurs through the urinary pathway after being primarily eliminated by biliary pathway, AFB1 was detected in different levels in kidney and urine (Polychronaki et al., 2008). Gupta and Sharma (2011) observed increased free radical generation during aflatoxicosis in kidney of mice fed $2 \mu \mathrm{g}$ AFB1/30 g body weight/day, which resulted in significant rise in thiobarbituric acid reactive substances (TBARS) along with a decrease in the activity of antioxidant enzymes (Gupta and Sharma, 2011). Similar adverse effects have been reported in kidney of mice fed $500 \mu \mathrm{g}$ AFB1/kg body weight/day, which resulted in the alteration of the oxidant/antioxidant balance leading to oxidative stress and significant histopathologic changes in the kidney tissue of the aflatoxin-treated group including hydropic and vacuolar degeneration, coagulation necrosis associated with degeneration of tubular architecture, as well as moderate parenchymal tubular degeneration (Eraslan et al., 2017). Similarly, another study also showed the pathological degeneration and necrosis in kidney of chickens fed aflatoxin at a concentration of $3 \mathrm{mg} / \mathrm{kg}$ diet (Gholami-Ahangaran et al., 2016).

Recently, many studies have focused on the scientific relevance of exploiting various agricultural by-products for alternative utilization as animal feed, including potential effects on health and animal performance (Ajila et al., 2012). Food and agricultural processing industry generate high levels of wastes that accumulate increasingly and exert negative effects on the environment (Azubuike et al., 2016; Girotto et al., 2015; Palade et al., 2019a). As a means of addressing this matter, food and agricultural waste management represents a solution for a better control and reduction of worldwide waste (Georganas et al., 2020; Ravindran and Jaiswal, 2016).

In this context, many plant by-products have been receiving supplementary attention towards the exploit of their bioactive compounds in view of nutritional utility for humans as well as for animals (Amagase, 2006; 
Galasso et al., 2016; Lee et al., 2017). Different studies reported that natural phytochemicals from various plant extracts and oils, as well as food components such as antioxidant compounds (phenolic compounds) may act effectively in alleviating the toxic effect of aflatoxins (Boudergue et al., 2017; Tulayakul et al., 2007).

Taking into account the proven beneficial applicability of food and agricultural by-products as feed additives in animal diets, recent scientific endeavours focus on their utility as mycotoxin binders in order to facilitate the decontamination of animal feed (Kolosova and Stroka, 2011). Grape seeds represent a potential source of valuable bioactive compounds, namely polyphenolics, which might be used as feed supplements rendering beneficial nutritional and health effects (Tagliazucchi et al., 2010). For instance, grape pomace has been reported as a new potentially efficient bio-sorbent for the removal of mycotoxins from liquid media (Avantaggiato et al., 2014). Similarly, grape seed cakes and grape seed meal (GSM) that are obtained from the oil extraction from grape seeds is a by-products rich in bioactive compounds, such as polyphenols as well as polyunsaturated fatty acids (PUFAs), which possess anti-inflammatory, anti-cancer, anti-microbial, antioxidative and immune-modulatory activities (Taranu et al., 2020a, 2020b). More recently, the successful evaluation of adsorption efficacy was investigated on AFB1, among other tested mycotoxins, using chitosan which mediated its removal from palm kernel cake (Abbasi Pirouz et al., 2020). Also, grape pomace was reported as an effective by-product in improving piglet physiology and gut health (Chedea et al., 2018; Taranu et al., 2018).

The aim of the present study was to assess the effects of dietary inclusion of grape seed meal on oxidative stress response in weaned piglets. Several markers of antioxidant status (antioxidant enzymes activity, total antioxidant status, and TBARS concentration) were measured in kidneys of weaned pigs in order to investigate the ability of GSM supplementation to mitigate the unwanted effects of aflatoxin B1 feed contamination, and to improve the antioxidant status.

\section{MATERIALS AND METHODS}

\section{Animals and experimental treatments}

Twenty-four cross-bred pigs (TOPIG-40) with an average body weight of $9.13 \pm 0.03 \mathrm{~kg}$ were randomly assigned to one of the four experimental diets: control diet (Control), aflatoxin B1 diet (diet contaminated with $320 \mu \mathrm{g}$ AFB1/kg feed), grape seed meal diet (diet with 8\% GSM) and AFB1+GSM group (320 $\mu \mathrm{g}$ AFB1/kg feed + 8\% GSM) for a period of 30 days. Animals were cared for in accordance with the Romanian Law 206/2004 and the EU Council Directive 98/58/EC for handling and protection of animals used for experimental purposes. 
The grape seed meal used for the GSM and GSM+AFB1 diets was obtained from S.C. OLEOMET-S.R.L., Bucuresti, Romania. The polyphenolic and polyunsaturated fatty acid (PUFA) profile of GSM and experimental feed was determined by LC-MS and GC, as previously reported by Taranu et al. (2019) (Taranu et al., 2019).

Diets containing AFB1 (AFB1 and GS+ AFB1 diets) were obtained by mixing $50 \mathrm{mg}$ of high purity AFB1 (FERMENTEC, Jerusalem, Israel), previously dissolved in DMSO (dimethyl sulfoxide), into conventional feed in order to achieve a concentration of $320 \mathrm{ppb}$ AFB1. The formulation of experimental diets followed the requirements for weaning feed as indicated by NRC (NRC, 2012), and was previously described by Taranu et al. (2019) (Taranu et al., 2019).

Animals were housed in pens, received experimental feed for 30 days, and were identified by ear tagging. At the end of the experimental period, the animals were euthanized and kidney samples were collected and washed with saline solution for blood removal. The samples were stored at $80^{\circ} \mathrm{C}$ until analyzed. The frozen organ samples were milled in liquid nitrogen.

\section{Mycotoxin analysis of diets}

Experimental feed ingredients were investigated for AFB1 initial contamination as previously reported by Taranu et al. (2019) (Taranu et al., 2019) and showed mycotoxin levels below the EU limits for pigs. The screening was performed by ELISA method using Veratox ELISA kits (Neogen, Lansing, MI, USA) according to the manufacturer's instructions, with the limit of detection (LOD) of $0.5 \mathrm{ppb}$, and the limit of quantification (LOQ) of 1-8 ppb. Aflatoxin B1 was also measured by UPLC (LOD- $0.008 \mathrm{ng} / \mathrm{ml}$ and LOQ-0.02 $\mathrm{ng} / \mathrm{ml}$, recovery rate, $96.7 \%$ ) after clean-up on immunoaffinity column, rendering a concentration of $320 \pm 10.9 \mathrm{ppb}$ in AFB1 diet and $2.4 \pm 0.15 \mathrm{ppb}$ in the Control diet, respectively.

\section{Evaluation of antioxidant status and lipid peroxidation}

The activities of antioxidant enzymes catalase (CAT), superoxide dismutase (SOD) and glutathione peroxidase (GPx) activities were measured using standardized kits (Cayman Chemical) according to the manufacturer's instructions as previously described by (Palade et al., 2019b). CAT activity was measured at $540 \mathrm{~nm}$. One unit was defined as the amount of enzyme that will cause the formation of $1.0 \mathrm{nmol}$ of formaldehyde per minute at $25^{\circ} \mathrm{C}$ and the results were expressed as $\mu \mathrm{mol} / \mathrm{min} / \mathrm{g}$ tissue. SOD activity is standardized using the cytochrome $\mathrm{c}$ and xanthine oxidase coupled assay. SOD activity was measured at 440-460 $\mathrm{nm}$ and the results were expressed as U/g tissue. One unit was defined as the amount of enzyme needed to exhibit 50\% dismutation of the superoxide radical. GPx activity was indirectly measured by a coupled 
reaction with glutathione reductase (GR) every minute at $340 \mathrm{~nm}$ (for $10 \mathrm{~min}$ ) and results were expressed as $\mu \mathrm{mol} / \mathrm{min} / \mathrm{g}$ tissue. One unit was defined as the amount of enzyme that will cause the oxidation of $1.0 \mathrm{nmol}$ of NADPH to $\mathrm{NADP}+$ per minute at $25^{\circ} \mathrm{C}$.

The antioxidant capacity of the kidney samples was measured with the TEAC (Trolox equivalent antioxidant capacity) assay using ABTS as radical cation (2,2'-azinobis-(3-ethylbenzothiazoline-6-sulphonic acid), a method previously described by (Chedea et al., 2019). Lipid peroxidation levels in kidney tissue was evaluated by measuring thiobarbituric acid-reactive substances (TBARS) as previously described by (Palade et al., 2019b).

\section{Statistical analysis}

All data are expressed as mean \pm standard error of the mean (SEM). All the results were submitted to SAS JMP software (SAS Analytics, USA) and. One-way analysis of variance (ANOVA) was performed to investigate the statistical differences between groups for all analyzed parameters employing Tukey's HSD (honestly significant difference) test. Additionally, data were analyzed as a $2 \times 2$ factorial design. The main effects included the diet type (with or without AFB1), grape seed meal supplementation (with or without GSM) and their interaction. Table 1 summarizes the information about the factors and their settings. Values of $\mathrm{P}<0.05$ were considered significant.

Table 1. Treatment groups and coded levels of factors

\begin{tabular}{|c|c|c|}
\hline Treatment group & \multicolumn{2}{|c|}{ Coded factor levels } \\
\hline & $\begin{array}{c}\text { Diet type } \\
\text { (with or without AFB1) }\end{array}$ & $\begin{array}{c}\text { GSM supplementation } \\
\text { (with or without GSM) }\end{array}$ \\
\hline Control & - & - \\
\hline AFB1 & + & - \\
\hline GSM & - & + \\
\hline AFB1 + GSM & + & + \\
\hline
\end{tabular}

\section{RESULTS AND DISCUSSION}

\section{Chemical composition of grape seed meal}

Grape seed meal, as a resulting by-product of grape seed oil extraction, are rich in polyphenolic compounds, among which tannins are contained in the highest amount (Marin et al., 2019). The inclusion of grape seed meal into the piglets' feed, as previously reported by Taranu et al. (2019) (Taranu et al., 2019), improved the overall chemical composition of the GSM experimental diet (increased crude fat and fiber contents). The polyunsaturated fatty acid (PUFA) profile revealed that PUFAs represented $65.16 \%$ of total FAMEs (fatty acid methyl esters) in grape seed meal, with $63.63 \%$ total $\omega-6$ and $1.54 \%$ total 
$\omega-3$. Linoleic acid was the most abundant $\omega-6$ FA (62.94\%), whereas alphalinolenic acid was the predominant $\omega-3$ fatty acid (0.85\%). Among the monounsaturated fatty acids (MUFA), oleic acid ( $\omega-9)$ was also present with a concentration of $15.30 \%$ (Taranu et al., 2020b, 2019).

In addition, the total polyphenol concentration and the antioxidant activity of GSM diet registered higher levels compared with those of the control. The polyphenol profile of GSM, expressed as catechin equivalents (CE), obtained through LC-MS analysis revealed a high content of phenolic acids such as caffeoilquinic acid $\left(57.4 \mu \mathrm{g} \mathrm{ml}^{-1} \mathrm{CE}\right)$, ferulic acid $(34.43 \mu \mathrm{g} \mathrm{ml}-1$ $\mathrm{CE})$, dicaffeoilquinic acid $\left(28.85 \mu \mathrm{g} \mathrm{ml}^{-1} \mathrm{CE}\right)$, along with procyanidin dimers (30.25 $\left.\mu \mathrm{g} \mathrm{ml}^{-1} \mathrm{CE}\right)$ and trimers $\left(13.02 \mu \mathrm{g} \mathrm{ml}{ }^{-1} \mathrm{CE}\right)$. In the flavan-3-ol class, catechin content was $13.61 \mu \mathrm{g} \mathrm{ml}^{-1} \mathrm{CE}$, while epigallocatechin was $22.28 \mu \mathrm{g}$ $\mathrm{ml}^{-1} \mathrm{CE}$ (Taranu et al., 2020b, 2019).

\section{Antioxidant defence status}

The used statistical model allows us to predict the response of analysed markers on the basis of model effects we prescribed, which is the full factorial effect between GSM and AFB1. The purpose herein was to assess the effect of GSM dietary inclusion on antioxidant status in kidney of piglets and also to see whether diets with or without AFB1 differ in that effect. Also, does AFB1 contamination alone predict differences in CAT/GPx/SOD/TAS/TBARS levels, regardless of GSM allocation? Accordingly, the interaction effect determines whether there is a different effect of GSM depending of AFB1; alternatively, whether there is a different effect of AFB1 depending on GSM.

Our results show that the lower levels of CAT activity, visible in the AFB1 fed group, are significantly improved by the dietary addition of GSM into the diet (Figure 1).

Catalase activity in kidney of weaned piglets was influenced by diet type (with or without AFB1, P $<0.0001^{*}$ ), whereas no significant effect was registered for GSM supplementation alone. However, the significant GSM $\mathrm{x}$ AFB1 interaction depicts that there is a different effect of GSM dietary inclusion depending on diet type (with or without AFB1). In other words, GSM alleviates the toxic effects of the toxin by increasing the CAT levels in AFB1 group, and maintains kidney CAT activity at physiological levels within the AFB1 unafflicted piglets.

Similar to CAT activity, pigs fed GSM + AFB1 exerted a higher SOD activity in kidneys compared with the AFB1 group (Figure 2). In addition, the interaction plot depicts the overall effect of GSM supplementation, which depends on diet type, that is with or without AFB1 (AFB1 x GSM, P $<0.0001 *$ ). It is worth noting the extent to which GSM dietary inclusion influences kidney SOD, resulting in no differences in healthy piglets, but higher levels in AFB1 fed group. 


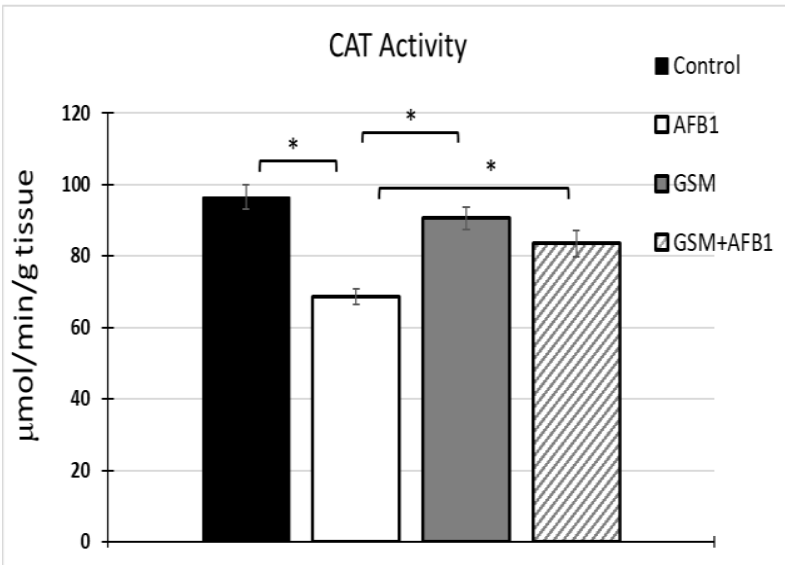

\begin{tabular}{lr} 
Source & Prob $>$ F \\
\hline AFB1 & $<.0001^{*}$ \\
GSM & 0.1704 \\
AFB1 x GSM & $0.0037^{*}$
\end{tabular}

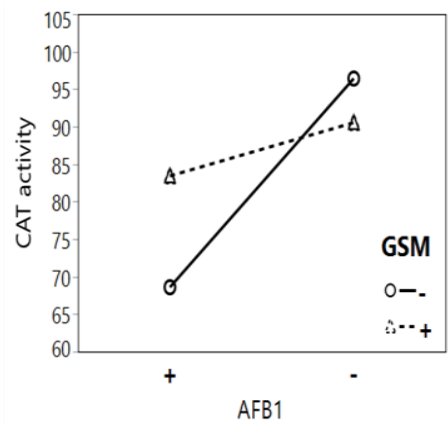

Figure 1. Effect of AFB1 exposure and GSM dietary supplementation on catalase activity (CAT) measured in kidney of weaned piglets. Data are means \pm SEM $(n=6)$. The “*” symbol indicates significant differences $(\mathrm{P}<0.05)$.

In this regard, the rate of dietary GSM inclusion is important, but for the rate used in this experiment there were no differences between the diet with GSM alone and the control diet. This effect could also be perceived in conjunction with CAT activity, indicating that the response to diet varies between the two groups (with or without AFB1), being more pronounced for CAT activity, and is further modulated by the addition of GSM.
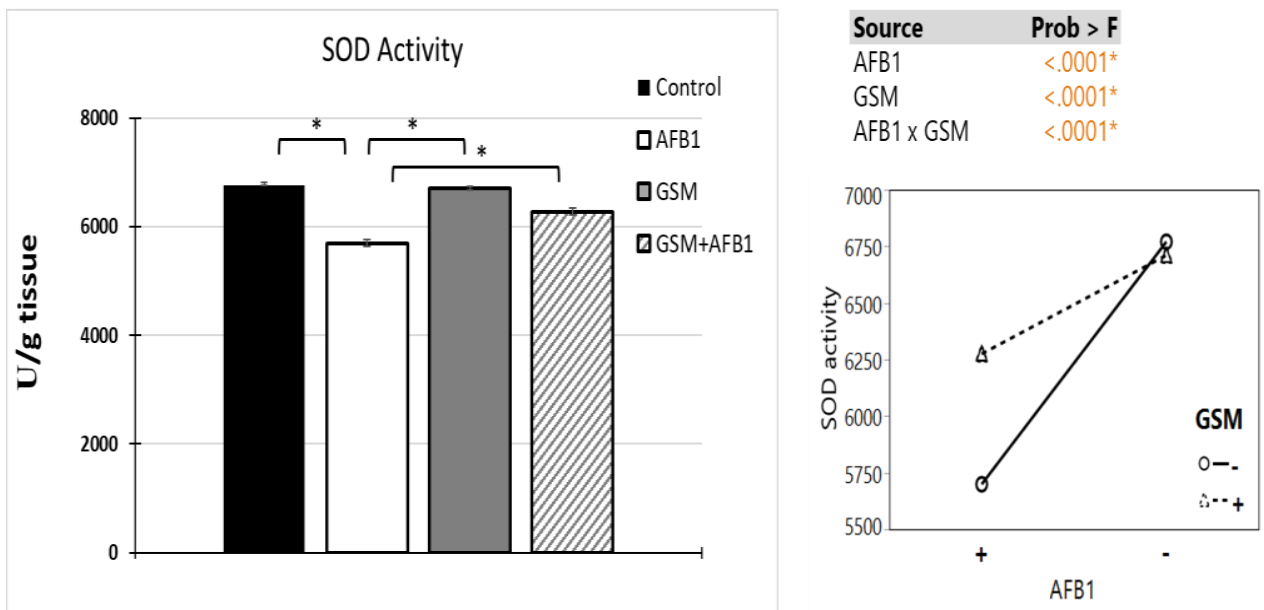

Figure 2. Effect of AFB1 exposure and GSM dietary supplementation on superoxide dismutase activity (SOD) measured in kidney of weaned piglets. Data are means \pm SEM $(n=6)$. The “*” symbol indicates significant differences $(P<0.05)$. 
Our study indicated that GSM supplied a higher content of PUFAs to the experimental diet. In this regard, PUFAs possess the capacity to improve upon the gene expression and activity of several antioxidant enzymes (Palade et al., 2019b). Accordingly, it can be inferred that GSM bioactive compounds (PUFAs and polyphenols) contributed to the improvement in antioxidant markers in a certain degree.

On the contrary, GPx activity was unaffected by AFB1 diet and by GSM supplementation (Figure 3). No significant interactions were observed for GPx activity. It could be perceived as the ability of the antioxidant defence system to maintain homeostasis by sustaining kidney GPx activity within the physiological levels and the attempt of the body to sustain redox balance at cellular level (Lubos et al., 2011; Sarıkaya and Doğan, 2020). This might suggest important implications of GSM constituents on the antioxidant defence system, which act by balancing out the negative effects of AFB1 contamination.

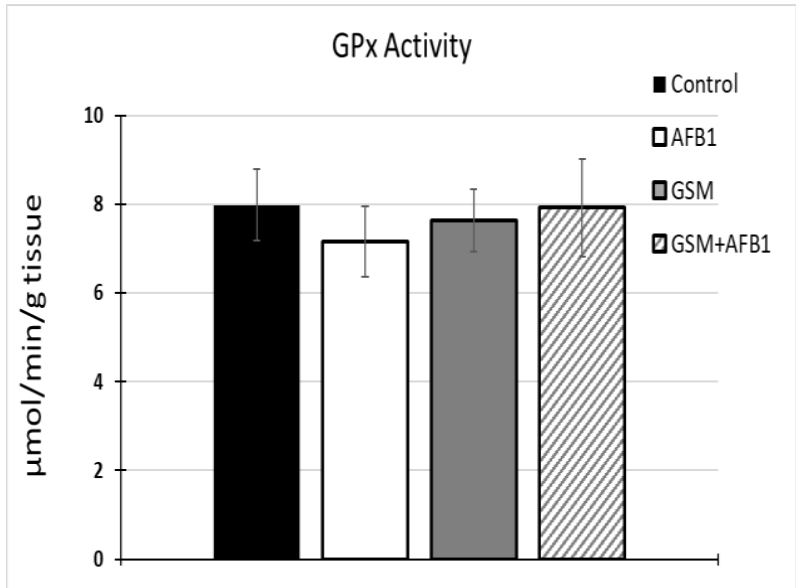

$\begin{array}{lr}\text { Source } & \text { Prob }>\text { F } \\ \text { AFB1 } & 0.3088 \\ \text { GSM } & 0.6903 \\ \text { AFB1 x GSM } & 0.5951\end{array}$

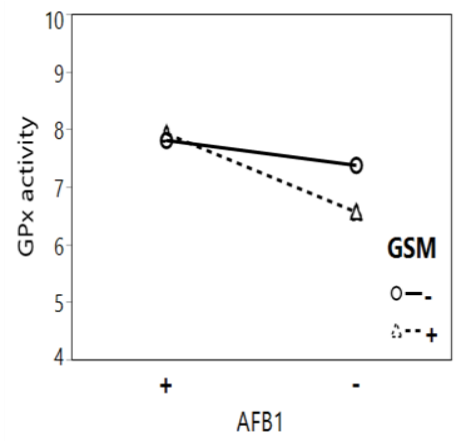

Figure 3. Effect of AFB1 exposure and GSM dietary supplementation on glutathione activity (GPx) measured in kidney of weaned piglets. Data are means \pm SEM $(n=6)$.

In our study, GSM dietary inclusion had no effect on TAS measured in kidney of weaned piglets (Figure 4), however, we observed a tendency for a significant interaction effect $(P=0.0772)$. Similar to $G P x$, it suggests a balanced interaction between the innate antioxidant system and GSM constituents.

In addition to the improvement in antioxidant enzymes, the total antioxidant status (TAS) in conjunction with the measurement of lipid peroxidation (TBARS) provide further information with regard to the overall oxidative status in kidney of AFB1 afflicted piglets. 

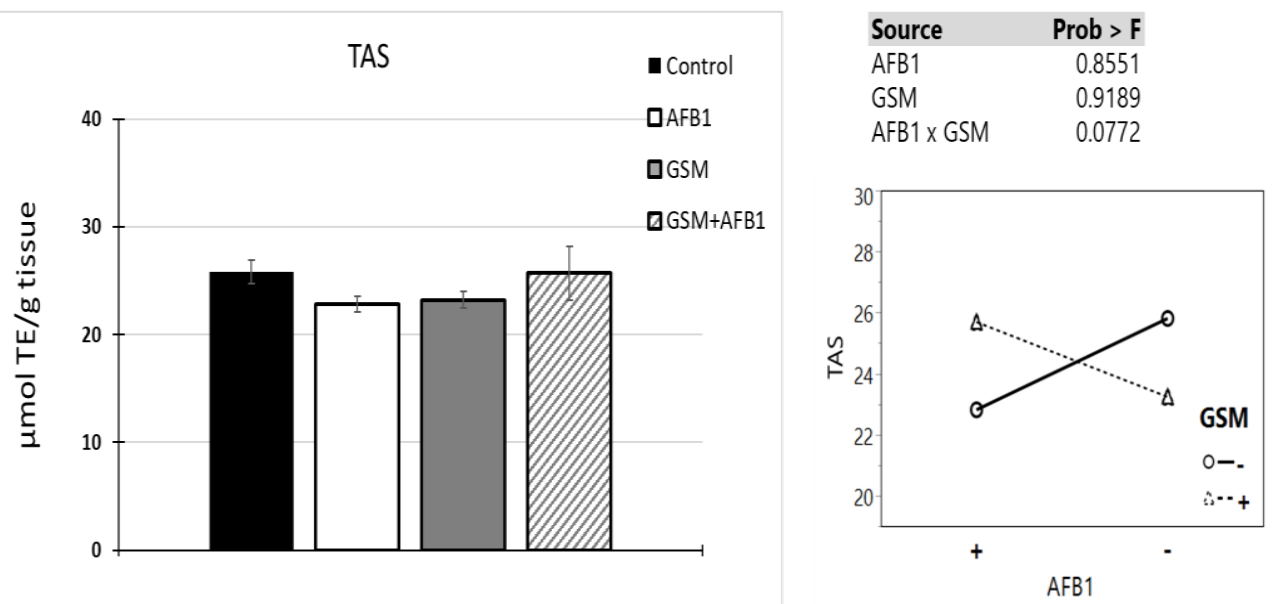

Figure 4. Effect of AFB1 exposure and GSM dietary supplementation on total antioxidant status (TAS) measured in kidney of weaned piglets. Data are means \pm SEM $(n=6)$.

The measurement of lipid peroxidation reveals increased TBARS levels in AFB1 fed group (Figure 5), which suggest that AFB1 lead to oxidative degradation of the lipids at membrane level and cell damage.

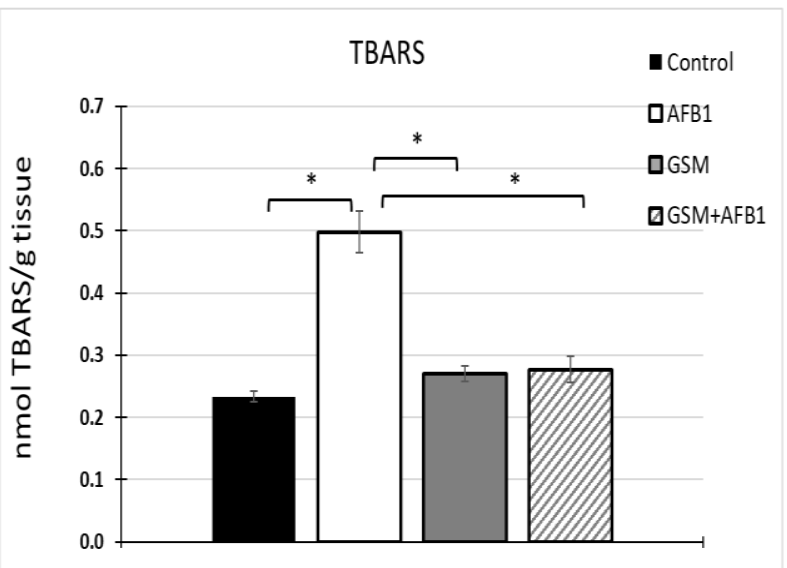

$\begin{array}{lr}\text { Source } & \text { Prob }>\text { F } \\ \text { AFB1 } & 0.0002^{*} \\ \text { GSM } & 0.0117^{*} \\ \text { AFB1 x GSM } & 0.0003^{*}\end{array}$

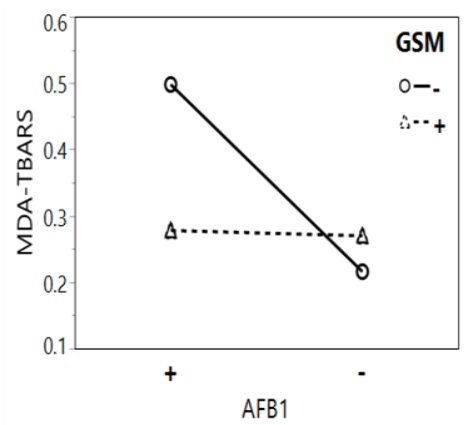

Figure 5. Effect of AFB1 exposure and GSM dietary supplementation on lipid peroxidation (TBARS) measured in kidney of weaned piglets. Data are means \pm SEM $(n=$ 6). The “*” symbol indicates significant differences $(\mathrm{P}<0.05)$.

Comparably, the addition of GSM in piglets' diet improved the oxidative status, evidenced by the significant interaction effect $\left(\mathrm{P}=0.0117^{*}\right)$. Accordingly, GSM supplementation alone registered differences in TBARS levels compared to AFB1, resulting in similar lipid peroxidation levels to those of the control group. Taking into account that TBARS test measures the lipid 
peroxidation products in its terminal phase, we believe that reactive oxygen species (ROS) have been generated in similar concentrations in the two aflatoxin groups (with and without AFB1), but the process of lipid peroxidation would have been inhibited in AFB1+GSM group due to the beneficial effect of GSM constituents, which in turn modulate the activity of the antioxidant enzymes.

In this study, we showed that the inclusion of GSM in the diet of weaned piglets influences the antioxidant status of AFB1 fed group by increasing the activity of antioxidant enzymes, CAT and SOD, as well as by decreasing the lipid peroxidation in kidney towards the values of the control group. On the other hand, we observed that GPx activity and TAS were not influenced by GSM diet. Nevertheless, the GSM dietary supplementation in weaned piglets had an overall positive influence on the antioxidant status markers in kidney of piglets.

Other studies reported similar in vitro and in vivo findings with regard to the influence of aflatoxicosis on kidney damage through oxidative stress (Eraslan et al., 2017; Karamkhani et al., 2020; Lei et al., 2013), as well as morphological tissue damage, and the efficacy of different bioactive compounds to alleviate these negative effects (Gholami-Ahangaran et al., 2016; Gupta and Sharma, 2011). For example, Li et a. (2018) provided insight on the involvement of L-proline, key metabolite of AFB1, in improving oxidative stress tolerance by being utilized in detoxifying kidney damage in a mice model ( $\mathrm{Li}$ et al., 2018). In another study, histopathological and biochemical changes induced by AFB1 in mice kidney also pointed out the induction of oxidative stress, which was subsequently alleviated by treatment with Tinospora cordifolia ethanolic extract (Gupta and Sharma, 2011).

Taken together, we observed that by administering dietary GSM to piglets exposed to AFB1, the significant increase of CAT and SOD activities and decrease of TBARS led to the inhibition of oxidative stress in kidney tissue, which suggests that GSM exerts a protective effect towards the toxic action of AFB1.

\section{CONCLUSION}

The findings of the present study revealed the positive effect of dietary addition of GSM on antioxidant enzyme activities (CAD and SOD) together with improved TBARS levels in kidney of weaned piglets. This is mainly evidenced by the elevated state of antioxidant defense system of AFB1+GSM group compared to the AFB1 group. Collectively, the results obtained in this study provide new insight into the beneficial effects of the dietary addition of GSM within the frame of sustainable animal production. In addition, taking into account the stability displayed by the other indicators of oxidative stress measured herein (GPx and TAS), the overall cause-effect relationship between 
the toxin and the added phytochemicals points to a better response of piglets receiving GSM to the kidney damage during aflatoxicosis.

\section{ACKNOWLEDGEMENTS}

This work was supported by funds from the National Research Projects 8PCCDI0473-PC1 granted by the Romanian Ministry of Research and Innovation.

\section{REFERENCES}

Abbasi Pirouz, A., Selamat, J., Zafar Iqbal, S., Iskandar Putra Samsudin, N., 2020. Efficient and Simultaneous Chitosan-Mediated Removal of 11 Mycotoxins from Palm Kernel Cake. Toxins (Basel). https://doi.org/10.3390/toxins12020115

Ajila, C.M., Brar, S.K., Verma, M., Tyagi, R.D., Godbout, S., Valéro, J.R., 2012. Bioprocessing of agro-byproducts to animal feed. Crit. Rev. Biotechnol. 32, 382-400. https://doi.org/10.3109/07388551.2012.659172

Amagase, H., 2006. Clarifying the real bioactive constituents of garlic. J. Nutr. 136, 716S-725S. https://doi.org/10.1093/jn/136.3.716S

Andretta, I., Kipper, M., Lehnen, C.R., Hauschild, L., Vale, M.M., Lovatto, P.A., 2012. Meta-analytical study of productive and nutritional interactions of mycotoxins in growing pigs. Animal 6, 1476-1482. https://doi.org/10.1017/S1751731111002278

Avantaggiato, G., Greco, D., Damascelli, A., Solfrizzo, M., Visconti, A., 2014. Assessment of Multi-mycotoxin Adsorption Efficacy of Grape Pomace. J. Agric. Food Chem. 62, 497-507. https://doi.org/10.1021/jf404179h

Azubuike, C.C., Chikere, C.B., Okpokwasili, G.C., 2016. Bioremediation techniques-classification based on site of application: principles, advantages, limitations and prospects. World J. Microbiol. Biotechnol. 32, 1-18. https://doi.org/10.1007/s11274-016-2137-X

Battilani, P., Toscano, P., Van der Fels-Klerx, H.J., Moretti, A., Camardo Leggieri, M., Brera, C., Rortais, A., Goumperis, T., Robinson, T., 2016. Aflatoxin B1 contamination in maize in Europe increases due to climate change. Sci. Rep. 6, 24328. https://doi.org/10.1038/srep24328

Boudergue, C., Burel, C., Dragacci, S., Favrot, M., Fremy, J., Massimi, C., Prigent, P., Debongnie, P., Pussemier, L., Boudra, H., Morgavi, D., Oswald, I., Perez, A., Avantaggiato, G., 2017. Review of mycotoxin-detoxifying agents used as feed additives: mode of action, efficacy and feed/food safety. EFSA Support. Publ. 6. https://doi.org/10.2903/sp.efsa.2009.en-22

Chedea, V.S., Palade, L.M., Marin, D.E., Pelmus, R.S., Habeanu, M., Rotar, M.C., Gras, M.A., Pistol, G.C., Taranu, I., 2018. Intestinal absorption and antioxidant activity of grape pomace polyphenols. Nutrients 10 . https://doi.org/10.3390/nu10050588

Chedea, V.S., Palade, L.M., Pelmus, R.S., Dragomir, C., Taranu, I., 2019. Red 
grape pomace rich in polyphenols diet increases the antioxidant status in key organs - kidneys, liver, and spleen of piglets. Animals 9. https://doi.org/10.3390/ani9040149

Colović, R., Puvača, N., Cheli, F., Avantaggiato, G., Greco, D., Đuragić, O., Kos, J., Pinotti, L., 2019. Decontamination of mycotoxin-contaminated feedstuffs and compound feed. Toxins (Basel). 11. https://doi.org/10.3390/toxins11110617

Eraslan, G., Sarıca, Z.S., Bayram, L.Ç., Tekeli, M.Y., Kanbur, M., Karabacak, M., 2017. The effects of diosmin on aflatoxin-induced liver and kidney damage. Environ. Sci. Pollut. Res. 24, 27931-27941. https://doi.org/10.1007/s11356-017-0232-7

Fox, E.M., Howlett, B.J., 2008. Secondary metabolism: regulation and role in fungal biology. Curr. Opin. Microbiol. 11, 481-487. https://doi.org/https://doi.org/10.1016/j.mib.2008.10.007

Galasso, I., Russo, R., Mapelli, S., Ponzoni, E., Brambilla, I.M., Battelli, G., Reggiani, R., 2016. Variability in Seed Traits in a Collection of Cannabis sativa L. Genotypes. Front. Plant Sci. 7, 20-25. https://doi.org/10.3389/fpls.2016.00688

Georganas, A., Giamouri, E., Pappas, A.C., Papadomichelakis, G., Galliou, F., Manios, T., Tsiplakou, E., Fegeros, K., Zervas, G., 2020. Bioactive compounds in food waste: A review on the transformation of food waste to animal feed. Foods 9, 1-18. https://doi.org/10.3390/foods9030291

Gholami-Ahangaran, M., Rangsaz, N., Azizi, S., 2016. Evaluation of turmeric (Curcuma longa) effect on biochemical and pathological parameters of liver and kidney in chicken aflatoxicosis. Pharm. Biol. 54, 780-787. https://doi.org/10.3109/13880209.2015.1080731

Girotto, F., Alibardi, L., Cossu, R., 2015. Food waste generation and industrial uses: A review. Waste Manag. 45, 32-41. https://doi.org/10.1016/j.wasman.2015.06.008

Gupta, R., Sharma, V., 2011. Ameliorative effects of tinospora cordifolia root extract on histopathological and biochemical changes induced by aflatoxin-b(1) in mice kidney. Toxicol. Int. 18, 94-98. https://doi.org/10.4103/0971-6580.84259

Karamkhani, M., Asilian-Mahabadi, H., Daraei, B., Seidkhani-Nahal, A., NooriZadeh, A., 2020. Liver and kidney serum profile abnormalities in workers exposed to aflatoxin B1 in urban solid waste management centers. Environ. Monit. Assess. 192, 472. https://doi.org/10.1007/s10661-02008422-y

Kolosova, A., Stroka, J., 2011. Substances for reduction of the contamination of feed by mycotoxins: a review. World Mycotoxin J. 4, 225-256. https://doi.org/10.3920/WMJ2011.1288

Kos, J., Janić Hajnal, E., Malachová, A., Steiner, D., Stranska, M., Krska, R., Poschmaier, B., Sulyok, M., 2020. Mycotoxins in maize harvested in 
Republic of Serbia in the period 2012-2015. Part 1: Regulated mycotoxins and its derivatives. Food Chem. 312, 126034. https://doi.org/https://doi.org/10.1016/j.foodchem.2019.126034

Kos, J., Mastilović, J., Hajnal, E.J., Šarić, B., 2013. Natural occurrence of aflatoxins in maize harvested in Serbia during 2009-2012. Food Control 34 , $31-34$ https://doi.org/https://doi.org/10.1016/j.foodcont.2013.04.004

Kosicki, R., Błajet-Kosicka, A., Grajewski, J., Twarużek, M., 2016. Multiannual mycotoxin survey in feed materials and feedingstuffs. Anim. Feed Sci. Technol. 215, $165-180$. https://doi.org/https://doi.org/10.1016/j.anifeedsci.2016.03.012

Lee, M.T., Lin, W.C., Yu, B., Lee, T.T., 2017. Antioxidant capacity of phytochemicals and their potential effects on oxidative status in animals - A review. Asian-Australasian J. Anim. Sci. 30, 299-308. https://doi.org/10.5713/ajas.16.0438

Lei, M., Zhang, N., Qi, D., 2013. In vitro investigation of individual and combined cytotoxic effects of aflatoxin B1 and other selected mycotoxins on the cell line porcine kidney 15. Exp. Toxicol. Pathol. 65, 1149-1157. https://doi.org/https://doi.org/10.1016/j.etp.2013.05.007

Li, H., Xing, L., Zhang, M., Wang, J., Zheng, N., 2018. The Toxic Effects of Aflatoxin B1 and Aflatoxin M1 on Kidney through Regulating L-Proline and Downstream Apoptosis. Biomed Res. Int. 2018. https://doi.org/10.1155/2018/9074861

Lubos, E., Loscalzo, J., Handy, D.E., 2011. Glutathione peroxidase-1 in health and disease: from molecular mechanisms to therapeutic opportunities. Antioxid. Redox Signal. 15, 1957-1997. https://doi.org/10.1089/ars.2010.3586

Marin, D.E., Bulgaru, C.V., Palade, L.M., Pistol, G.C., Gras, M.A., Taranu, I., 2019. Effect of the grape seed meal administration on inflammation and oxidative stress in the spleen of piglets fed aflatoxin B1. Arch. Zootech. $22,22-31$.

NRC, 2012. Nutrient Requirements of Swine: Eleventh Revised Edition. The National Academies Press, Washington, DC. https://doi.org/10.17226/13298

Palade, L.M., Croitoru, C., Arnous, A., 2019a. Preliminary assessment for the synthesis of lignin-type molecules using crude onion peroxidase. Chem. Pap. 73, 801-810. https://doi.org/10.1007/s11696-018-0651-z

Palade, L.M., Habeanu, M., Marin, D.E., Chedea, V.S., Pistol, G.C., Grosu, I.A., Gheorghe, A., Ropota, M., Taranu, I., 2019b. Effect of dietary hemp seed on oxidative status in sows during late gestation and lactation and their of spring. Animals 9, 1-19. https://doi.org/10.3390/ani9040194

Peles, F., Sipos, P., Győri, Z., Pfliegler, W.P., Giacometti, F., Serraino, A., Pagliuca, G., Gazzotti, T., Pócsi, I., 2019. Adverse Effects, Transformation and 
Channeling of Aflatoxins Into Food Raw Materials in Livestock. Front. Microbiol. 10, 1-26. https://doi.org/10.3389/fmicb.2019.02861

Polychronaki, N., Wild, C.P., Mykkänen, H., Amra, H., Abdel-Wahhab, M., Sylla, A., Diallo, M., El-Nezami, H., Turner, P.C., 2008. Urinary biomarkers of aflatoxin exposure in young children from Egypt and Guinea. Food Chem. Toxicol. 46 ,

519-526.

https://doi.org/https://doi.org/10.1016/j.fct.2007.08.034

Prapapanpong, J., Udomkusonsri, P., Mahavorasirikul, W., Choochuay, S., Tansakul, N., 2019. In vitro studies on gastrointestinal monogastric and avian models to evaluate the binding efficacy of mycotoxin adsorbents by liquid chromatography-tandem mass spectrometry. J. Adv. Vet. Anim. Res. 6, 125-132. https://doi.org/10.5455/javar.2019.f322

Ravindran, R., Jaiswal, A.K., 2016. Exploitation of Food Industry Waste for High-Value Products. Trends Biotechnol. 34, 58-69. https://doi.org/https://doi.org/10.1016/j.tibtech.2015.10.008

Sarıkaya, E., Doğan, S., 2020. Glutathione Peroxidase in Health and Diseases, in: Bagatini, M.D. (Ed.), Glutathione System and Oxidative Stress in Health and Disease. IntechOpen, Rijeka, p. Ch. 3. https://doi.org/10.5772/intechopen.91009

Tagliazucchi, D., Verzelloni, E., Bertolini, D., Conte, A., 2010. In vitro bioaccessibility and antioxidant activity of grape polyphenols. Food Chem. 120, 599-606. https://doi.org/10.1016/j.foodchem.2009.10.030

Taranu, I., Gras, M.A., Habeanu, M., Pistol, C., Lefter, N., Palade, M.L., Chedea, V.S., Marin, D.E., 2020a. Active ingredients from oil by-products modulate spleen inflammatory and antioxidant response in pigs. Arch. Zootech. ica 23, 81-97. https://doi.org/10.2478/azibna-2020-0008

Taranu, I., Habeanu, M., Gras, M.A., Pistol, G.C., Lefter, N., Palade, M., Ropota, M., Sanda Chedea, V., Marin, D.E., 2018. Assessment of the effect of grape seed cake inclusion in the diet of healthy fattening-finishing pigs. J. Anim. Physiol. Anim. Nutr. (Berl). 1-13. https://doi.org/10.1111/jpn.12697

Taranu, I., Hermenean, A., Bulgaru, C., Pistol, G.C., Ciceu, A., Grosu, I.A., Marin, D.E., 2020b. Diet containing grape seed meal by-product counteracts AFB1 toxicity in liver of pig after weaning. Ecotoxicol. Environ. Saf. 203, 110899. https://doi.org/10.1016/j.ecoenv.2020.110899

Taranu, I., Marin, D.E., Palade, M., Pistol, G.C., Chedea, V.S., Gras, M.A., Rotar, C., 2019. Assessment of the efficacy of a grape seed waste in counteracting the changes induced by aflatoxin B1 contaminated diet in performance, plasma, liver and intestinal tissues of pigs after weaning. Toxicon 162, 24-31. https://doi.org/https://doi.org/10.1016/j.toxicon.2019.02.020

Tulayakul, P., Dong, K.S., Li, J.Y., Manabe, N., Kumagai, S., 2007. The effect of feeding piglets with the diet containing green tea extracts or coumarin on in vitro metabolism of aflatoxin B1 by their tissues. Toxicon 50, 339-348. https://doi.org/https://doi.org/10.1016/j.toxicon.2007.04.005 